\title{
PAPER
}

\section{Discrete and dynamic scaling of the size of continuous graphic movements of parkinsonian patients and elderly controls}

\author{
M G Longstaff, P R Mahant, M A Stacy, A W A Van Gemmert, B C Leis, G E Stelmach
}

J Neurol Neurosurg Psychiatry 2003;74:299-304

See end of article for authors' affiliations

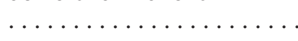

Correspondence to: Dr M G Longstaff, Motor Control Laboratory, Arizona State University, PO Box 870404, Tempe, AZ 85287-0404, USA; mitchell.longstaff@asu.edu

Received 19 April 2002

In revised form

23 October 2002

Accepted

28 November 2002

Objectives: To systematically investigate the ability of Parkinson's disease patients to discretely and dynamically scale the size of continuous movements and to assess the impact of movement size on outcome variability.

Methods: Ten patients with Parkinson's disease (mean age 72 years) were compared with 12 healthy elderly controls (mean age 70 years). The subjects wrote with a stylus on a graphics tablet. In experiment 1 they drew circles, matching the size of five target circles ranging in magnitude from a radius of $0.5 \mathrm{~cm}$ up to $2.5 \mathrm{~cm}$. In experiment 2 they drew spirals with a radius of at least $2 \mathrm{~cm}$. In both experiments the drawings were initially performed as accurately as possible then as fast and accurately as possible.

Results: In both experiments the patients and controls drew at a similar speed. The within trial variability of the pen trajectory was greater for patients than controls, and increased disproportionately with the size of the movement. When the emphasis was on size rather than variability (circles), the patients' drawing movements were the same size as controls. When the emphasis was on accuracy of pen trajectory (that is, minimum variability) rather than size (spirals), the patients' drawing movements were smaller than controls.

Conclusions: The movements made by Parkinson's disease patients are hypometric partly as an adaptive strategy used to reduce movement variability. This strategy is used primarily when the requirement to make accurate movements outweighs the need to make large movements.

$\mathrm{P}$ arkinson's disease (PD) patients commonly experience deterioration in their ability to perform well learned motor tasks such as handwriting and aiming movements. ${ }^{12}$ Typical symptoms include a tendency to produce movements that are slower (bradykinesia), smaller (hypometric), and more variable than elderly controls. ${ }^{3}$ While these symptoms are sometimes observed in experimental situations, ${ }^{4-6} \mathrm{PD}$ patients are frequently able to increase the size and speed of their movements as required by the experimental task, often matching the magnitudes produced by elderly controls. ${ }^{7-9}$ It remains unclear why PD patients produce hypometric movements when they have the capacity to increase movement size.

This study examines the relation between the size and speed of a movement and the resulting variability, using tasks that involve steady movements and a range of sizes. The specific aim was to systematically investigate the ability of PD patients to scale the size of a continuous movement and to examine the impact of movement size on outcome variability. Outcome variability relates to the difference between the task requirements and the actual movements.

These issues were investigated by examining the drawing movements of PD patients and elderly controls under several conditions. Circles of increasing size were drawn to see how variability changes as size increases. In this case the size of the movement is emphasised. A second task (spiral drawing) involving a similar movement was used, however in this case the shape of the movement is emphasised while the subjects are free to choose a preferred size.

These two fine motor tasks require accuracy during either discrete (circles) or continuous and steady ( spiral) changes in the magnitude of the movement. This study extends research that has focused on discrete tasks or discrete scaling of movement magnitude. ${ }^{20}{ }^{11}$ The spiral task has a particular appeal as

it entails an accurate movement that requires a continuous change in size. As such, it displays features of common motor skills such as handwriting. Furthermore, previous studies have used spiral drawing as a qualitative neurological assessment tool for the assessment and understanding of motor control disorders such as PD. ${ }^{12-18}$ However there is a paucity of empirical studies designed to elucidate the mechanisms involved in the task. Past research indicates that a healthy person who does not display signs of motor dysfunction and who can perform this task with a high degree of accuracy will produce "smooth" curves when drawing a spiral. Those who suffer from motor function degradation will produce less smooth "wobbly" curves when drawing a spiral. ${ }^{13}$

If patients have a deficit in their ability to increase the magnitude of their movements, they will produce movements that are smaller than that produced by controls. If, however, they are able to meet task demands of size and speed in a similar manner to elderly controls, then their primary deficit lay elsewhere. It could be that the observed impairments are not attributable to an inability to produce movements of a particular size and speed, but that size and/or speed reduction is an adaptive strategy used to minimise unwanted variability.

Indeed, several studies have suggested that there is not so much a deficit in the ability to generate the force required for movements of a particular size, but that the forces used (and therefore the movements) are more variable. ${ }^{6}{ }^{19}{ }^{20}$ For example, Stelmach $e a^{21}$ report that PD patients performed similarly to controls in a force production task, which implies that they have an accurate "internal model" of the required forces. However, the patients had more irregular force-time curves, suggesting a more "noisy" output from the motor system and an inability to produce smooth forces. It has also been reported that, in general, force variability increases with the force produced. ${ }^{22}$ 
Table 1 Summary of dominant symptoms of the Parkinson's disease patients*

\begin{tabular}{|c|c|c|c|c|c|c|c|c|c|}
\hline $\mathrm{N}$ & Age (y) & Sex & $\begin{array}{l}\text { Diagnosed onset } \\
\text { (y) }\end{array}$ & Micrographia & $\begin{array}{l}\text { Tremor at } \\
\text { rest }\end{array}$ & Rigidity & Bradykinesia & $\begin{array}{l}\text { Hoehn and } \\
\text { Yahr stage }\end{array}$ & Medication \\
\hline 1 & 77 & Male & 10 & Yes & No & No & Yes & 2.5 & $\mathrm{SCR}, \mathrm{M}, \mathrm{Ar}$ \\
\hline 2 & 59 & Male & $<1$ & Yes & Yes & Yes & Yes & 3 & $N / A$ \\
\hline 3 & 71 & Male & 4 & Yes & Yes & Yes & No & 2 & SCR \\
\hline 4 & 77 & Male & 18 & Yes & Yes & Yes & Yes & 3 & $\mathrm{Ar}$ \\
\hline 5 & 81 & Male & 2 & Yes & Yes & Yes & No & 2 & $\mathrm{SR}$ \\
\hline 6 & 71 & Female & 7 & Yes & Yes & Yes & Yes & 3 & SCR \\
\hline 7 & 76 & Female & 6 & Yes & No & Yes & Yes & 3 & $\mathrm{SCR}, \mathrm{E}$ \\
\hline 8 & 66 & Male & 5 & No & Yes & Yes & No & 2 & SR \\
\hline 9 & 63 & Male & 3 & Yes & No & No & Yes & 2.5 & SCR \\
\hline 10 & 74 & Female & 20 & Yes & No & No & Yes & 3 & $S R, M$ \\
\hline
\end{tabular}

*All PD diagnoses were made by the patients' neurologist. As symptomology can vary within and between patients, dominant symptoms present immediately before testing were assessed using the UPDRS subscale iii: Motor examination. The Hoehn and Yahr ${ }^{27}$ assessment of stages was also performed. While all subjects exhibited at last some body bradykinesia and rigidity, they are only included as dominant symptoms if they were rated higher than 1 (on a 0-4 scale) on the UPDRS. Tremor at rest was only included if it occurred in the arm used to perform the experiment. Micrographia was determined after asking subjects about changes in their handwriting. SSCR, sinemet CR; SR, sinemet regular; M, mirapex; E, eldepryl; Ar, artane. Subject 2 was recently diagnosed and had not begun taking medication.

One confounding factor is that often the movements produced by patients are smaller or slower than that produced by controls, so movement variability is not always directly comparable. To determine if group differences are related to size and/or speed of movement, target movements of a range of sizes and at two different speeds were investigated. Typically, movement speed also increases along with the length of the radius, a relation that has been described as the $2 / 3$ power law. $^{23}$

\section{EXPERIMENT 1: SCALING OF SIZE WHILE DRAWING CIRCLES}

This experiment investigates two aspects of motor control. The first is the possibility that PD patients have a deficit in their ability to produce movements of a specified size as the magnitude of the required movement increases. This is tested by examining the ability of each group to discretely increase the length of the radius in line with an increase in target size. If patients have a deficit in their ability to produce movements of a given size, they will produce smaller movements than controls. If size and speed are reduced as a strategy to minimise trajectory variability, then these parameters will not be adjusted when trajectory variability is de-emphasised and target size and movement speed has been emphasised. As evidence suggests that patients have the capacity to scale their movements, ${ }^{89}$ it is hypothesised that they will draw circles that match the size and speed of those drawn by elderly controls.

The second aspect of motor control tested is the notion that movement variability tends to increase along with the size of the movement. This is partly because of the necessity to involve more joints to complete the task. As the size of the circle radius increases, more joints (fingers, then fingers/wrist and finally fingers/wrist/arm) become actively involved. ${ }^{24}$ Past research has found that movement variability of multi joint movements is greater in PD patients, ${ }^{24}$ leading to the conclusion that $\mathrm{PD}$ patients have deficits in the coordination of multiple joints. ${ }^{1125}$

This, along with the knowledge that force variability is greater in parkinsonian patients, ${ }^{1926}$ leads to the hypothesis that PD patients have a deficit in their ability to produce smooth and accurate movements, with their performance increasingly deteriorating as the magnitude of their movements increases. It is predicted that PD patients will draw circles with a greater degree of variability compared with elderly controls. In accordance with the relation between the size and speed of drawing movements ${ }^{23}$ it is expected that the larger circles will be drawn faster than the smaller circles. Increasing the size of the circle also increases the coordination requirements. It is therefore predicted that larger circles will be drawn with a greater degree of variability than the smaller circles.

\section{Methods}

Subjects

Subjects were 10 PD patients between the ages of 59 and 81 years ( seven men, three women; mean age $=72$ ) and 12 healthy elderly controls between the ages of 62 and 82 years ( six men, six women; mean age $=70$ ). All subjects were right handed. The patients were recruited from the Muhammad Ali Parkinson Research Center, Barrow Neurological Institute, Phoenix, Arizona under the direction of Dr M Stacy. Patients were tested at the institute with the cooperation of their neurologist. They were all bilaterally impaired idiopathic PD patients and were tested after an overnight fast of at least 12 hours from their last intake of PD medication (see table 1 for an account of their dominant symptoms). The elderly controls were recruited from a volunteer database and received a small payment to compensate them for travel expenses. All subjects signed informed consent forms.

\section{Apparatus}

The subjects performed the circle drawing task with a conventional style inking pen on a blank sheet of white paper. The paper was fixed to the surface of a digitiser tablet (Wacom Intuos $12 \times 18$ ) so that the drawing movements were performed directly in front of the subjects. The digitiser tablet recorded the $\mathrm{X}$ and $\mathrm{Y}$ position of the tip of the pen with a sampling frequency of $206 \mathrm{~Hz}$ and a spatial resolution of $0.001 \mathrm{~cm}$. The experiment was controlled, and the data recorded, by an OASIS $^{28}$ macro running on a laptop computer. A sheet of paper on which the target circles were presented was affixed at the top of the digitiser in a position so that the subjects could see them at all times.

\section{Procedure}

To compare the results, experiments 1 and 2 were performed consecutively in the same experimental session. This minimises confounding factors such as the variability in symptoms that can occur within patients over time. Graphic movements such a circle and spiral drawing are considered to be over-learned skills ${ }^{29}$ as they are comparatively simple tasks that have been performed many times previously. As such, any training effects would relate to familiarisation with the procedures. To reduce any possible training effects, the tasks were design to be as natural as possible. This was achieved by the use of a stylus that was similar to a typical ballpoint pen, and the writing surface was a piece of paper attached to the top of the digitiser. Subjects were allowed to adopt a comfortable 


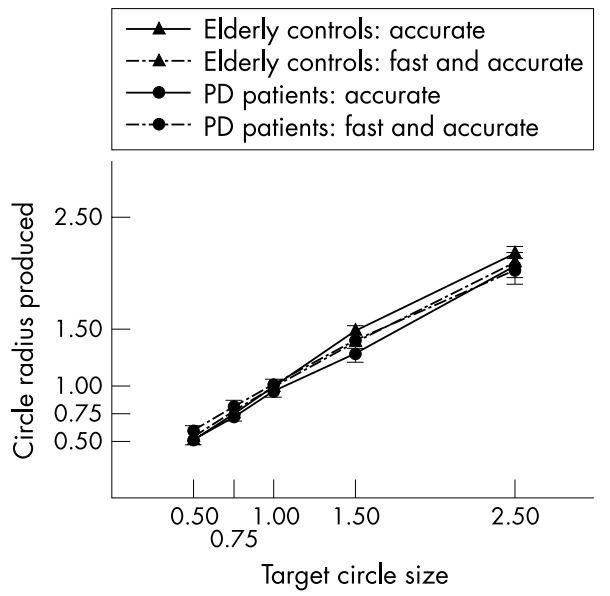

Figure 1 Circle radius produced for each target circle size (vertical bars indicate standard error).

posture and could lean on the digitiser. The tasks were explained and demonstrated to the subjects who then practised them until they were comfortable with what was required and the experimenter determined that they understood the task. There were several short rest periods during each experiment, and a longer rest period between experiments to prevent fatigue.

Testing occurred between 830 am and $1200 \mathrm{pm}$ and took about 30 minutes for each patient. The subjects were asked to draw several revolutions each of five different sized circles ( 0.5 $\mathrm{cm}, 0.75 \mathrm{~cm}, 1 \mathrm{~cm}, 1.5 \mathrm{~cm}$, and $2.5 \mathrm{~cm}$ ). They were asked to match the size of the target circle and to continue drawing until they heard a tone produced by the computer. This tone sounded after five revolutions had been drawn. The task was repeated twice, resulting in three trials of each circle size. Initially, the subjects were free to draw the circles at a comfortable speed. The task was then repeated, however in this condition the participants were required to draw the circles as fast as possible. In both conditions, an emphasis was placed on producing an accurate circle shape and size.

\section{Data analysis}

The raw coordinate data were filtered with a seven point median filter to reduce measurement error. The mean and variance of the radius of each circle was determined. A measure of trajectory variability around an ideal circle was computed by dividing the variance of the radius by the radius length for each trial. This enabled a direct comparison of the proportional variability for different circle sizes. The mean tangential velocity (TV) each subject used to draw the circles was also calculated. These means were entered into a general linear model (GLM) procedure, according to a completely factorial design with participants nested within groups; two groups (elderly controls; PD patients) $\times$ two accuracy conditions (accurate; fast and accurate) $\times$ five circle size conditions $(0.5,0.75,1,1.5$, and $2.5 \mathrm{~cm})$. A significance level of 0.05 was used for all analyses.

\section{Results}

\section{Circle size}

Figure 1 shows the magnitude of the circles produced by the subjects for each target circle size. There was no significant main effect of group, with both groups drawing each target circle at about the same magnitude. There was a significant main effect for circle size, $F(4,80)=492.969$, p $<0.05$, with larger target circles being drawn with larger radius. From figure 1 it can be seen that the radii produced for the smaller circles were close to the target size (means of 0.54, 0.75, and 0.98). However, the subjects increasingly undershot the magnitude as the target size increased (means of 1.38 and 2.1).

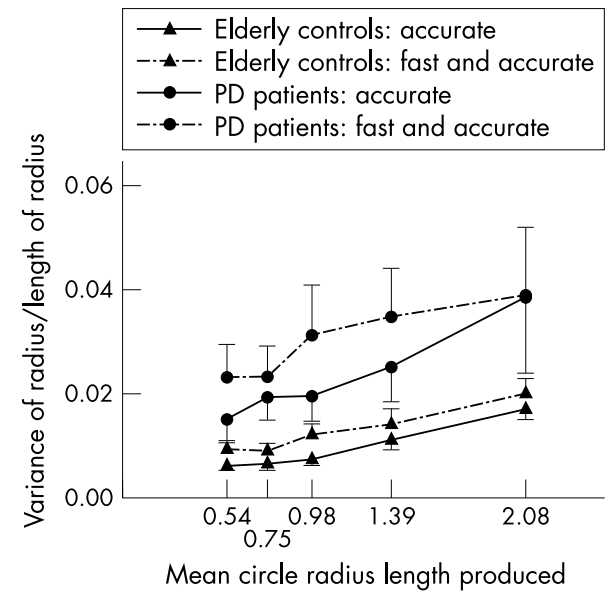

Figure 2 Proportional variability (variance of radius divided by length of radius) for each circle size produced (vertical bars indicate standard error).

There was a significant condition by circle size interaction, $F(4,80)=3.857, \mathrm{p}<0.05$, resulting from a slightly larger radius for the a small circles under the fast and accurate condition but no difference between the radii from the two conditions for the mid to large circles. There was no significant effect of speed condition and no other significant interactions. It is evident that there was a consistent tendency to undershoot the size of the larger target circles. This pattern of results suggest that there is a linear scaling of the magnitude of the radius, but this scaling parameter is set too low to achieve the target circle size for the larger circles.

\section{Proportional variability}

The mean proportional variability for each circle, for each group and each condition can be seen in figure 2. Patients were found to draw circles with more than twice as much variability (0.027) compared with controls $(0.011), F(1,20)=4.836$, $\mathrm{p}<0.05$. There was a significant main effect for circle size, $F(4,80)=9.393, \mathrm{p}<0.05$, with larger circles being drawn with greater variability $(0.013,0.014,0.018,0.021,0.022$, from smallest to largest). There was a greater degree of variability when drawing the circles in the fast and accurate condition (0.022) compared with the accurate condition (0.017), $F(1,20)=12.855, \mathrm{p}<0.05$. There were no significant interactions. These results indicate that trajectory variability is greater for patients, and that the variability for both groups is amplified as the size and speed of the movement increases.

\section{Tangential velocity}

The tangential velocity each group used to draw the circles in both conditions can be seen in figure 3. The GLM procedure revealed a significant main effect for circle size, $F(4$, $80)=87.243, p<0.05$, with larger circles being drawn faster than smaller circles (from smallest to largest circles $T V=3.9$, $4.9,5.7,6.8$, and $8.7 \mathrm{~cm} / \mathrm{s})$. The circles were drawn about half as fast in the accurate condition $(4.0 \mathrm{~cm} / \mathrm{s})$ compared with the fast and accurate condition $(7.9 \mathrm{~cm} / \mathrm{s}), F(1,20)=75.215$, $\mathrm{p}<0.05$.

There was a significant condition by circle size interaction, $F(4,80)=8.763, \mathrm{p}<0.05$, because of a greater increase in TV from the smallest to the largest circle in the fast and accurate condition $(6.03 \mathrm{~cm} / \mathrm{s}$ increase $)$ compared with the accurate condition $(3.49 \mathrm{~cm} / \mathrm{s}$ increase). There were no other significant interactions and no significant group effect. In terms of changes in magnitude of the TV, the principal result was that larger circles were drawn faster, and that circles were drawn faster in the fast and accurate condition. 


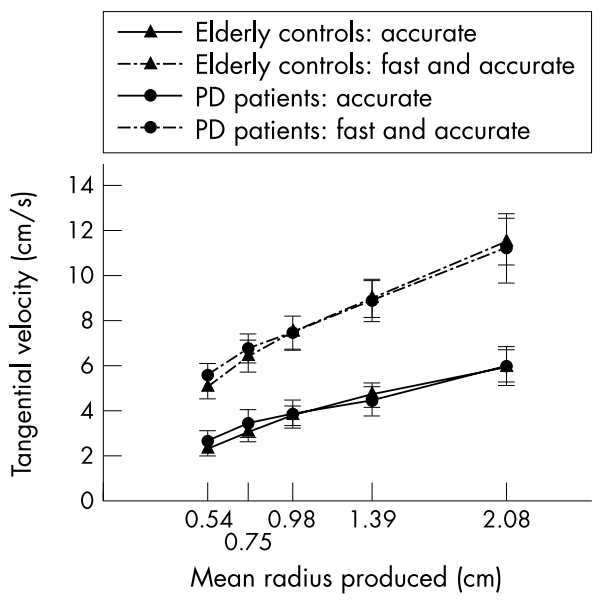

Figure 3 Tangenital velocity of drawing movements for each circle size produced (vertical bars indicate standard error).

\section{EXPERIMENT 2 SCALING OF SIZE WHILE DRAWING SPIRALS}

Experiment 1 demonstrated that PD patients have the capacity to increase the size and speed of their movements in a similar way to elderly controls. The patients also drew each circle with a greater degree of variability than controls. As the size and speed of the movement increased the proportional variability of the movement increased. This implies that an effective strategy to decrease variability would be to reduce the size and/or the speed of the movement. That movement speed is reduced as an adaptive motor strategy has been previously suggested. ${ }^{29}$ However, we propose that reducing movement size might also be an effective strategy. As variability increased more with size than speed in this task, the most effective strategy would be to reduce the size of the movement.

To test this argument a task was selected (spiral drawing) that contained movements similar to those involved in circle drawing. However, this time the shape of the resulting pen trace was of greater importance and the participants had the freedom to choose how they scaled the size and speed of the movement. The only restrictions on this was that they were to continue drawing until the radius of the spiral was greater than $2 \mathrm{~cm}$ and in the fast condition they had to draw faster. This task requires a high degree of spatial accuracy to be performed well.

In this experiment the ability of each group to continuously increase the length of the radius while increasing the speed at which they move their pen was examined. It was expected that both groups would be able to steadily increase the speed and magnitude of their movements. If patients use a strategy of reducing the size of their movements to reduce variability, then their spirals will be globally smaller than that drawn by controls. If the patients use a strategy of reducing the speed of their movements to reduce variability, then spirals of the same size will be drawn slower for patients. Alternatively, the spirals may be drawn smaller and slower. If size and/or speed reduction is not an adaptive strategy to minimise variability, then the subjects will draw spirals at a similar size and speed to controls. As the results of experiment 1 suggest that size reduction is a more effective strategy, it is hypothesised that PD patients will use a smaller scaling ratio (radius/degrees) when drawing the spiral, resulting in a smaller, but stable, distance between each successive revolution. It is expected that patients will draw the spirals as fast as controls.

Finally, it is expected that patients will display a greater level of variability around an ideal trajectory when compared with elderly controls. The task was initially performed with an emphasis on accuracy of shape. A fast and accurate condition was also included to examine the influence of speed on trajectory variability in spiral drawing.

\section{Method}

Subjects and apparatus

The subjects and apparatus used were the same as in experiment 1, however the target circles were removed. An example of a spiral was provided on a separate sheet of paper.

\section{Procedure}

The subjects were asked to draw a spiral, an example of which was demonstrated to them by the experimenter. They were asked to continue until they heard a tone produced by the computer. This tone sounded when the spiral radius was at least $2 \mathrm{~cm}$ and they had produced at least four revolutions. They were free to draw the spiral at a comfortable speed, in either direction (clockwise or anti-clockwise), and with a distance between each revolution of their own choosing. They were told however that once chosen they were to maintain the distance between each revolution at that level as accurately as possible. This is a requirement for producing a perfect spiral. This was repeated five times, resulting in a total of six spirals. Emphasis was placed on the accuracy of spiral shape and the consistency of the distance between each revolution. The subjects were then asked to draw another six spirals, however this time they were instructed to draw as fast and accurately as possible.

\section{Data analysis}

All 10 PD patients and 10 of the 12 elderly controls naturally chose to draw their spirals in an anti-clockwise direction while the other two controls varied the direction from trial to trial. It was therefore decided to exclude the data from these two subjects from further analysis. The raw position data were converted into polar coordinates (that is, radius and angle of revolution). The radius was calculated from the starting position of the pen and the angle of revolution was a measure of how many revolutions the pen had travelled around this starting point. The definition of an ideal spiral centred at the origin can be expressed in polar coordinates as $r=a \theta$, where $r=x^{2}+y^{2}, \theta$ is the angle of revolution and $a$ is a constant. This results in a linear relation between angle of revolution and radius length. If the task requirements were met, regression analysis would result in a perfectly linear relation between angle of revolution (the independent variable) and radius length (the dependent variable).

The amount of variance explained by the regression equation is informative about the magnitude of the perpendicular deviations around the ideal trajectory. Low values indicate a greater difficulty in maintaining the accuracy of the local trajectory. The slope of the regression line is a measure of how "compact" the spiral is. The greater the slope, the greater the distance between each revolution, and the larger the global size of the spiral for a given number of revolutions.

The mean $r^{2}$ values and slope of the linear regression line were determined. These means were entered into a GLM procedure, according to a completely factorial design with participants nested within groups; two groups (elderly controls; PD patients) $\times$ two accuracy conditions (accurate; fast and accurate). The spirals were split into two sections for the calculation of the tangential velocity. The inner section included pen movements up to a radius of $1 \mathrm{~cm}$ and the outer section included movements from 1 to $2 \mathrm{~cm}$. This enables movements of the same magnitude to be compared between individuals as well as the small and large movements made by each individual. The mean TVs were entered into a GLM procedure, according to a completely factorial design with participants nested within groups; two groups (elderly controls; PD patients) $\times$ two accuracy conditions (accurate; fast and accurate) $\times$ two spiral sections (inner; outer).

\section{Results}

Trajectory variability

There was a significant difference between groups $F(1,18)=15.792, \mathrm{p}<0.05$, with controls drawing spirals with 
less variability around the ideal trajectory than patients $\left(r^{2}=0.83\right.$ compared with 0.71$)$. The generally high $r^{2}$ values reveal that both groups could perform the task reasonably well and were scaling the magnitude in a generally linear fashion. There were no significant main effects of condition and no condition by group interaction.

\section{Scaling of the magnitude of the spiral radius}

A significant main effect was found for group, $F(1,18)=6.438$, $\mathrm{p}<0.05$, with the controls drawing spirals with a greater distance between each revolution compared with patients (5.2 $\mathrm{mm}$ per revolution compared with $3.0 \mathrm{~mm}$ per revolution). There was no significant main effect of experimental condition and no condition by group interactions.

\section{Tangential velocity of the pen tip}

There was a significant main effect for experimental condition $F(1,18)=69.559, \mathrm{p}<0.05$, with the subjects drawing spirals more than twice as fast in the fast and accurate condition $(6.5 \mathrm{~cm} / \mathrm{s})$ compared with the accurate condition $(2.9 \mathrm{~cm} / \mathrm{s})$. There was also a significant main effect for spiral section, $F(1$, $24)=48.924, \mathrm{p}<0.05$, due to the larger outer spirals being drawn with a higher TV $(6.0 \mathrm{~cm} / \mathrm{s})$ than the smaller inner sections $(3.4 \mathrm{~cm} / \mathrm{s})$. There was a significant condition by spiral section interaction, $F(1,18)=38.168, \mathrm{p}<0.05$, with a much greater increase in speed from the inner to outer sections for the fast and accurate condition (a $3.9 \mathrm{~cm} / \mathrm{s}$ increase) compared with the accurate condition (a $1.3 \mathrm{~cm} / \mathrm{s}$ increase). There was no significant main effect of group, nor were there any other significant interactions. Overall, these results indicate that both groups drew the spirals at a similar speed that increased in the fast and accurate condition and as the movements became larger.

\section{DISCUSSION}

This study investigated the ability of PD patients to scale the size and speed of drawing movements and the impact that this has on movement variability. In experiment 1 subjects were asked to produce a continuous movement with discrete scaling. The results confirm that both groups were able to increase their movement speed in a similar way when drawing circles of varying sizes, with both groups increasing their speed in the "fast and accurate" condition. Furthermore, the results support the contention that PD patients would draw circles the same size as the controls, increasing the size of their movements along with the target circles.

The hypothesis that PD patients would draw circles with a greater degree of variability was also supported by the results. The proportional variability was higher for PD patients, and increased along with circle size for both groups. This means that the impact of variability on the shape of the circle increases disproportionately along with the size of the circle. The larger the movement, the greater the effect of variability. This variability leads to circles that look "messy" and less round. The variability was slightly higher in the fast and accurate condition but this difference was small compared with the increase in variability as size increased, as well as the differences between the groups. The results suggest that because movement size was the predominant requirement, the patients increased the size of their movements while tolerating a more variable trajectory and a less than perfect circle shape.

In experiment 2, the subjects were given the freedom to choose a scaling magnitude. As was predicted, PD patients used a smaller scaling ratio when drawing spirals (that is, they increased the magnitude of their movements at a slower rate), compared with elderly controls. However, there was no deficit in their ability to increase the size of their drawing movements up to a radius of $2 \mathrm{~cm}$. In accordance with the hypothesis, patients produced spirals with a greater degree of variability around the ideal trajectory compared with elderly controls. There was no change in variability in the fast and accurate condition.

Patients drew the spirals at a similar speed to that of elderly controls with both groups drawing the spirals faster in the "fast and accurate" condition. This increase in speed was most pronounced in the larger outer sections of the spirals, which were generally drawn faster than the smaller inner sections. These results are consistent with the findings of Van Gemmert et al, ${ }^{11}$ who found evidence that patients could scale speed in a similar way to elderly controls, but had difficulty in controlling movement amplitude, when they had to discretely scale the size of a handwriting-like movement. Finally, both groups could increase the speed at which they draw spirals as the magnitude of the radius increases and as the task conditions demanded.

Taken together, these results suggest that the PD patients were not impaired in their ability to scale the amplitude or speed of their fine motor movements. However, there was a substantial divergence in the quality of their movements, as evidenced by a greater degree of variability in the movements of PD patients compared with elderly controls. This heightened variability is not attributable to differences in the size or speed at which the two groups performed the task. Instead, this study revealed a more subtle difference between the two groups. When the subjects had the freedom to choose how they scaled their movements, patients tended to be use a smaller scaling ratio, making the increase in magnitude more gradual. This produced spirals that were globally smaller for a given number of revolutions, resulting in a more compact appearance (that is, a smaller distance between the spiral revolutions).

These results suggest that a smaller scaling ratio is selected as an effective strategy to minimise movement variability. Patients start with a level of variability that is already higher than elderly controls; any increase in size will produce a corresponding disproportionate increase in variability. By only increasing the magnitude of the movement a small amount for each revolution of the spiral, the subject is only increasing the variability by a small amount. This low variability is critical for meeting the task requirements.

It could be maintained that the findings reflect the fact that the patients exhibited typical symptoms such as bradykinesia, rigidity, and tremor. Rigidity, for example, could result in smaller movements as the range of movement related EMG might be reduced because of increased muscle co-activation in PD patients. However, the results show that the patients had no difficulty increasing the speed or size of their movements, producing global movements at a similar magnitude to elderly controls. This would imply that the range of movement related EMG tested in this study was the same for both elderly controls and PD patients, even if the patients had higher levels of co-contraction. Therefore, these symptoms did not impose undue constraint on the execution of task related movements. Furthermore, in a review article on bradykinesia in PD, Berardelli et al ${ }^{31}$ write that there is little evidence that rigidity is a limiting factor in $\mathrm{PD}$ movements, concluding that the role of rigidity has yet to be proved conclusively.

These are several possible mechanisms for heightened movement variability in PD patients and the relation between movement size and variability. For example, the variability might be directly related to the production and maintenance of muscle forces. A number of studies have shown that PD patients are able to specify particular forces, but the variability of the forces generated is much greater than controls. ${ }^{6} 192026$ Furthermore, force variability generally increases along with the force produced. ${ }^{22}$ Larger and faster movements require higher (and therefore, more variable) force levels. A second potential mechanism relates to the coordination of multiple joints. It has been documented that as the number of joints actively utilised increases, so too does movement variability, and that this increase is generally greater for 
PD patients than controls. ${ }^{24}$ As the size of the required movement increases, more joints become actively involved, increasing the coordination demands on the CNS.

This finding could partly explain why patients sometimes produce smaller movements than elderly controls. An example is the common symptom of micrographia in the handwriting of people with PD. Handwriting is a continuous task that requires online scaling of loop-like movements. It is a skill where the accuracy of letter shape is of utmost importance. There is no benefit to producing letters of a particular size if the writing is so messy that it is illegible. Given the choice between accuracy and size, the patients may try to maximise accuracy even though their resulting movements are still more variable than elderly controls. While this is speculative, it is supported by a study of parkinsonian handwriting by Rogers et al. ${ }^{32}$ Through the use of visual cues they were able to increase the size of the handwriting of PD patients. However, this was accompanied by a marked disturbance in word construction, including errors of letter formation and word orientation. Further research will investigate if this motor strategy is a general behaviour or mainly used for these types of hand movements.

It seems then, that while the patients are able to produce movements of a given size, they choose not to. Their ability to scale movements seems to be relatively intact, but the quality of their movements is inferior. There is a trade off between the necessity of producing the required size and producing the movement smoothly and accurately. When movement size is the predominant requirement, $\mathrm{PD}$ patients are satisfied with a more variable trajectory. However, when given the freedom to choose a scaling magnitude and an emphasis on accuracy of the shape of the trajectory, they produce smaller movements.

\section{ACKNOWLEDGEMENTS}

This research is supported by a national institute of neurological disorders and stroke grant, NS33173. Portions of this study were presented at the 10th biennial conference of the International Graphonomics Society, Nijmegen, 2001 and the 27th Annual Society For Neuroscience Conference, San Diego, 2001.

\section{Authors' affiliations}

M G Longstaff, A W A Van Gemmert, B C Leis, G E Stelmach, Motor Control Laboratory, Department of Kinesiology, Arizona State University, Tempe, Arizona, USA

P R Mahant M A Stacy, Muhammad Ali Parkinson Research Center, Barrow Neurological Group, Phoenix, Arizona, USA

Competing interests: none declared.

\section{REFERENCES}

1 Contreras-Vidal JL, Teulings HL, et al. Micrographia in Parkinson's disease. NeuroReport 1995;6:2089-92.

2 Rand MK, Stelmach GE, Bloedal JR. Movement accuracy constraints in Parkinson's disease patients. Neuropsychologia 2000;38:203-12.

3 Phillips JG, Stelmach GE, Teasdale, N. What can indices of handwriting quality tell us about parkinsonian handwriting? Hum Mov Sci 1991; 10:301-14.

4 Longstaff MG, Mahant PR, Stacy MA, et al. Continuously scaling a continuous movement: Parkinsonian patients choose a smaller scaling ratio and produce more variable movements compared to elderly controls. In: Meulenbroek RGJ, Steenbergen B, eds. Proceedings of the 10th Biennial Conference of the International Graphonomics Society. Niimegen: IGS, 2001

5 Van Gemmert AWA, Adler CH, Stelmach GE. The isochronic size range is reduced in handwriting of Parkinsonian patients. In:
Meulenbroek RGJ, Steenbergen B, eds. Proceedings of the 10th Biennial Conference of the International Graphonomics Society. Nijmegen: IGS 2001.

6 Vinter A, Gras P. Spatial features of angular drawing movements in Parkinson's disease patients. Acta Psychol 1998;100:177-93.

7 Oliveira RM, Gurd JM, Nixon P, et al. Micrographia in Parkinson's disease: the effect of providing external cues. J Neurol Neurosurg Psychiatry 1997; 63:429-33.

8 Teulings HL, Stelmach GE. Control of stroke size, peak acceleration, and stroke duration in Parkinsonian handwriting. Hum Mov Sci $1991 ; 10: 315-34$

9 Van Gemmert AWA, Teulings HL, Stelmach GE. The influence of mental and motor load on handwriting movements in Parkinsonian patients. Acta Psycholog 1998;100:161-75.

10 Dounskaia N, Van Gemmert AWA, Stelmach GE. Interjoint coordination during handwriting-like movements. Exp Brain Res 2000;135: 127-40

11 Van Gemmert AWA, Teulings HL, Contreras-Vidal JL, et al. Parkinson's disease and the control of size and speed in handwriting. Neuropsychologia 1999;37:685-94.

12 Alusi SH, Worthington J, Glickman S, et al. Evaluation of three different ways of assessing tremor in multiple sclerosis. J Neurol Neurosurg Psychiatry 2000;68:756-60.

13 Bain PG, Findley L. Assessing tremor severity: a clinical handbook. London: Smith-Gordon, 1993.

14 Bain PG, Findley L, Atchison P, et al. Assessing tremor severity. J Neurol Neurosurg Psychiatry 1993;56:868-73.

15 Longstaff MG. A theoretical and empirical investigation of the performance and clinical diagnostic value of handwriting and other graphic skills. [PhD thesis.] Newcastle: University of Newcastle, 2000.

16 Longstaff MG, Heath RA. The influence of tremor on handwriting performance under conditions of low and intermediate physical stress. Journal of Forensic Document Examination 2000;13:25-44.

17 Louis ED, Barnes LF, Wendt KJ, et al. Validity and test-retest reliability of a disability questionnaire for essential tremor. Mov Disord 2000;15:516-23.

18 Pullman SL. Spiral analysis: a new technique for measuring tremor with a digitizing tablet. Mov Disord 1998;13:85-9.

19 Stelmach GE, Castiello U. Functional force control in Parkinsons disease patients. In: Stelmach GE, Requin J, eds. Tutorials in motor behaviour II. Amsterdam: North-Holland, 1992.

20 Stelmach GE, Worringham CJ. The preparation and production of isometric force in Parkinson's disease. Neuropsychologia 1988;26:93-103.

21 Stelmach GE, Teasdale N, Phillips J, et al. Force production characteristics in Parkinson's disease. Exp Brain Res 1989;76:65-72.

22 Sherwood DE, Schmidt RA. The relationship between force and force variability in minimal and near-maximal static and dynamic contractions. Mot Behav 1980;12:75-89.

23 Lacquaniti F, Terzuolo C, Viviani P. The law relating the kinematic and figural aspects of drawing movements. Acta Psychol 1983;54:1 15-30.

24 Teulings HL, Contreras-Vidal JL, Stelmach GE, et al. Coordination of fingers, wrist and arm in Parkinsonian handwriting. Exp Neurol 1997; 146:159-70.

25 Berardelli A, Accornero N, Argenta M, et al. Fast complex arm movements in Parkinson's disease. J Neurol Neurosurg Psychiatry 1986:49:1146-9.

26 Vaillancourt DE, Slifkin AB, Newell KM. Intermittency in the visual control of force in Parkinson's disease. Exp Brain Res 2001;138:11827

27 Hoehn MM, Yahr MD. Parkinsonism: onset, progression, and mortality. Neurology 1967; 17:427-42.

28 De Jong WP, Hulstijn W, Kosterman BJM, et al. OASIS software and its application in experimental handwriting research. In: Simner ML, Leedham CG, Thomassen AJWM, eds. Handwriting and drawing research: basic and applied issues. Amsterdam: IOS Press, 1996.

29 Braun C, Heinz U, Schweizer R, et al. Dynamic organization of the somatosensory cortex induced by motor activity. Brain 2001; 124:2259-67.

30 Martin KE, Phillips JG, lansek R, et al. Inaccuracy and instability of sequential movements in Parkinson's disease. Exp Brain Res 1994; 102:131-40.

31 Berardelli A, Rothwell JC, Thompson PD, et al. Pathophysiology of bradykinesia in Parkinson's disease. Brain 2001;124:2131-46.

32 Rogers DK, Eddy MS, Morris ME, et al. The effects of visual cues on micrographic handwriting in Parkinson's disease. In: Meulenbroek RGJ, Steenbergen B, eds. Proceedings of the 10th Biennial Conference of the International Graphonomics Society. Nijmegen: IGS, 2001. 